\title{
The Cauchy Problem for Hyperbolic Equations with Double Characteristics
}

By

\author{
Nobuhisa IWASAKI*
}

\section{\$0. Introduction}

In the last decade, the theory on the Cauchy problem for hyperbolic equations has developed a little in the analysis of their characters. This article will aim to survey briefly the main point of the advance. Before doing so, we give a short historical remark. The notion of hyperbolic equation began from the characterization of the wave equation. In the present day, however, it comes to be understood as an algebraic and geometric characterization, for symbols of partial differential operators, corresponding to solvability of non characteristic Cauchy problem for them with data of a suitable function space, so called "well posed". Especially it seems to have been considered that the solvability to the space of infinitely differentiable functions called $\mathscr{E}$-well posed is essential since J. Hadamar [5] proposed. In fact I. G. Petrowsky [24] and completely L. Garding [8] characterized the necessary and sufficient condition for it to be $\mathscr{E}$ wcll posed in case of constant coefficients. In general, namely, to the equations with variable coefficients, P. D. Lax [14] and S. Mizohata [19] got a necessary condition that the principal part of the equations should be hyperbolic if the Cauchy problem for it is well posed. Nevertheless the generic fact known about the sufficient condition invariant under the change of variables had been essentially only one given by I. G. Petrowsky [25] for a long time. The fact is that the Cauchy problem for a hyperbolic equation with simple characteristics, called strictly hyperbolic, is $\mathscr{E}$-well posed, although it was extended until uniformly symmetrizable ones in case of systems of operators. The case of constant coefficients and the results by A. Lax [13] and by M. Yamaguti [28] have indicated the necessity of clarifying relations between the principal part and the lower

Received January 17, 1983.

* Rescarch Institute for Mathematical Sciences, Kyoto University. Kyoto 606, Iapan. 
order terms of operator at the singular points of characteristics. In fact, S. Mizohata and Y. Ohya [21] and [22] show, in the case of characteristics with constant multiplicity two, that the Levi condition, connected with the E. E. Levi's work [15], is necessary and sufficient to be $\mathscr{E}$-well posed, where the Levi condition is that the subprincipal part vanishes identically on the characteristics. This relation is extended in succession by $\mathrm{H}$. Flaschka and G. Strang (necessity) [4] and by J. Chazarain (sufficiency) [1] for operators with heigher constant multiplicities, noted by S. Matsuura [17] to be multi-products of distinct strictly hyperbolic operators. It means that such an operator satisfies the intrisically same type of conditions as ones in case of constant coefficients given by $\mathrm{L}$. Hörmander [6] and justified by L. Svensson [26]. Namely the condition is that the lower order terms are weaker than the principal part. (After them, J. L. Dunn (sufficiency) [3] and S. Wakabayashi (necessity) [27] have checked it valid even for the lower order terms with variable coefficients.) However there existed examples studied by M. Chi [2], O. A. Oleinik [23] and others which had different types of relations from ones supposed in case of constant coefficients. It were V. Ya Ivrii and V. M. Petkov [9] who went through these points to get a necessary condition advanced further. Their results refined by $\mathrm{L}$. Hörmander [7] were sharp as enough as a sufficient condition, better than the Petrowsky condition, was conjectured. We here summarize them and sufficient conditions by V. Ya Ivriǐ, L. Hörmander and the author. One of the conclusions for single equations is that the strong hyperbolicity is equivalent to the effective hyperbolicity.

Remark. Throughout this paper, symbols of pseudodifferential operators are the Weyl symbols. An operator $q(x, D)$ with the symbol $q(x, \xi)$ is defined by

$$
q(x, D) \phi=(2 \pi)^{-n} \int e^{i(x-y) \xi} q((x+y) / 2, \xi) \phi(y) d x d \xi .
$$

\section{§1. Necessary Conditions}

We consider a single partial differential operator $p$ of order $m$ on an open set $\Omega$ of $\boldsymbol{R}^{n+1}$. The non characteristic Cauchy problem, in other words, the initial value problem, is to find a solution of the equation $p u=f$ on $\Omega$ satisfying the initial data on a hypersurface of the derivatives up to $m-1$ of $u$ to the conormal direction of the surface. The solvability of the Cauchy problem according to J. Hadamard [5] consists of two parts of local existence of solutions and local 
uniqueness. There are some variants to realize it in exact notions. We use here one of stronger definitions in the sense that it is an open condition, namely, if it is solvable at a point, then it is solvable at any point of a neighborhood of it.

Definition 1.1. The Cauchy problem for $p$ is said to be well posed at a point $x^{\sim}$ with respect to a non characteristic direction $\theta \neq 0$ if there exist a neighborhood $\Omega$ of $x^{\sim}$ and an infinitely differentiable function $\phi$ satisfying $\phi\left(x^{\sim}\right)=0$ and $d \phi\left(x^{\sim}\right)=\theta$ such that the following statements $(E)_{t}$ and $(U)_{t}$ hold for any small $t$. $(E)_{t}$ For every $f$ belonging to $C_{0}^{+\infty}(\Omega)$ there is a distribution $u$ belonging to $\mathscr{E}^{\prime}(\Omega)$ and satisfying the equation $p u=f$ on $\Omega_{t}$, where $\Omega_{t}$ is the set of $x$ in $\Omega$ such that $\phi(x)<t$.

$(U)_{t}$ If $u$ belonging to $\mathscr{E}^{\prime}(\Omega)$ satisfies $p u=0$ on $\Omega_{t}$, then $u$ vanishes identically on $\Omega_{t}$.

By P. D. Lax [14] and S. Mizohata [19], we have known that the principal part $p_{m}$ of $p$ is hyperbolic if the Cauchy problem for $p$ is well posed as follows. (It is proved under weaker conditions of well-posedness.)

Theorem 1.1 (P. D. Lax and S. Mizohata). If the Cauchy problem for $p$ is well posed at $x^{\sim}$ with respect to the direction $\theta \neq 0$ such that $p_{m}\left(x^{\sim}, \theta\right) \neq 0$, then

$$
p_{m}(x, \zeta \theta+\xi)=0
$$

has only real roots in $\zeta$ for any real vector $\xi$ and for any $x$ of a neighborhood of $x^{\sim}$.

We can find in L. Hörmander [7] the above result to imply that

$$
p_{m(\beta)}^{(\alpha)}\left(x^{\sim}, \zeta^{\sim} \theta+\xi^{\sim}\right)=0
$$

for any derivatives in $(\xi, x)$ of order $(\alpha, \beta)$ up to $|\alpha|+|\beta|<r$ if

$$
p_{m}\left(x^{\sim}, \zeta \theta+\xi \sim\right)=0
$$

has the roots $\zeta^{\sim}$ of multiplicity $r$. So we have the following theorem by $\mathrm{V}$. Ya Ivriǐ and V. M. Petkov [9].

Theorem 1.2 (V. Ya Ivrii and V. M. Petkov). Suppose that $p_{m}\left(x^{\sim}, \zeta \theta+\xi^{\sim}\right)$ $=0$ has the real root $\zeta^{\sim}$ of multiplicity $r$. If the Cauchy problem for $p$ is well posed at $x^{\sim}$ with respect to the direction $\theta \neq 0\left(p_{m}\left(x^{\sim}, \theta\right) \neq 0\right)$ and has the finite propagation property, then it holds for the lower order term $p_{s}$ of order $s$ that

$$
p_{s(\beta)}^{(\alpha)}\left(x^{\sim} \cdot \zeta^{\sim} \theta+\xi^{\sim}\right)=0
$$


for any derivative in $(\zeta, x)$ of order $(\alpha, \beta)$ up to

$$
|\alpha|+|\beta|+2(m-s)<r .
$$

Remark. The finite propagation property means a stronger condition for the uniqueness such that $u$ belonging to $\mathscr{E}^{\prime}(\Omega)$ and satisfying $p u=0$ on $\Omega(\varepsilon, x)$ should vanish on $\Omega(\varepsilon, x)$, for any small and positive $\varepsilon$ and for any $x$ in $\Omega$, where $\Omega(\varepsilon, x)$ is the set of $x^{\prime}$ in $\Omega$ such that $\phi(x)-\phi\left(x^{\prime}\right)>\varepsilon\left|x-x^{\prime}\right|$.

Therefore we can define the localized operator of $p$.

Definition 1.2. Let $p_{l o c}$ be called the localization of $p$ if it is well defined for any point $\left(x^{\sim}, \xi^{\sim}\right)$ such that

$$
p_{\text {loc }}(x, \xi)=\lim _{v \rightarrow+s} v^{-2 m+r} p\left(x^{\sim}+v^{-1} x, r^{2}\left(\xi^{\sim}+v^{-1} \xi\right)\right)
$$

at the $r$-ple characteristic point $\left(x^{\sim}, \xi^{\sim}\right)$ of $p_{m}$.

Remark. The case of double characteristics does not need the results of the above theorem.

At the non characteristic point of $p_{m}, p_{l o c}$ is non zcro constant. At the simple characteristic point, it defines a non singular vector field of real constant coefficients. These two cases have no other information from the well-posedness of the Cauchy problem for $p$. At the double characteristic point, $p_{l o c}$ is a polynomial in $(x, \xi)$ of order 2 . If we denote the homogeneous part in $(x, \xi)$ of order $j$ by $p_{\text {loc }(j)}$, then $p_{\text {Ioc( } 2)}$ is defined from the Hesse matrix of $p_{m}, p_{\text {loc }(1)}$ is identically zero and $p_{l o c(0)}$ is equal to $p_{m-1}\left(x^{-}, \zeta^{\sim}\right) . \quad p_{l o c(2)}$ is characterized by the fundamental (Hamilton) matrix $\mathscr{F}$ defined as

$$
\sigma(X, \mathscr{F} X)=p_{\text {lor (2) }},
$$

where $X=(x, \zeta)$ and $\sigma$ is the canonical two form $\sum d \xi^{i} \wedge d x_{j}$. The hyperbolicity yields the properties of the fundamental matrix $\mathscr{F}$ that the eigenvalues of $\mathscr{F}$ are only on the pure real and pure imaginary axes and the non zero real eigenvalues, if they exist, are only one by one on the positive part and the negative part of the real axis, respectively. If we define the positive trace $\operatorname{Tr}^{\sim} \mathscr{F}$ of $\mathscr{F}$ by

$$
\operatorname{Tr}^{\sim} \mathscr{F}=2^{-1} \sum\left|\operatorname{Im} \lambda_{j}\right|,
$$

where $\lambda_{j}$ are all eigenvalues of $\mathscr{F}$, then we are able to conclude the following.

Theorem 1.3 (V. Ya Ivriǐ. V. M. Petkov and L. Hörmander). The subprincipal part $p_{m-1}$ should be real and hounded as 


$$
-2^{-1} \operatorname{Tr}^{-\mathscr{F}} \leq p_{m-1} \leq 2^{-1} \operatorname{Tr}^{\sim} \mathscr{\mathscr { F }}
$$

by the positive trace of the fundamental matrix $\mathscr{F}$ on the singular points of the characteristic set where the fundamental matrix had no non zero real eigenulue if the Cauchy problem for $p$ were well posed.

Remarks. 1) The multiplicities of the singular points need not be always double because the subprincipal part $p_{m-1}$ should be zero by virtue of the previous theorem while the fundamental matrix is zero at the point where the multiplicity is not less than triple. V. Ya Ivrii and V. M. Petkov have remarked that the finite propagation property is not necessary to obtain this conclusion.

2) We may also call the inequality at the above theorem the Levi condition.

Now we introduce two notations.

Definition 1.3. 1) Let $p_{m}$ be hyperbolic with respect to the direction $\theta \neq 0$. $p_{m}$ is said effectively hyperbolic (with respect to the direction $\theta \neq 0$ ) if the fundamental matrix at any singular point of $p_{m}$ has non zero real eigenvalues.

2) $p_{m}$ is said strongly hyperbolic (with respect to the direction $0 \neq 0$ ) if the Cauchy problem for $p$ with any arbitrary lower order term is well posed (with respect to the direction $0 \neq 0$ ).

Corollary 1.4 (V. Ya Ivrií and V. M. Petkov). If $p_{m}$ is strongly hyperbolic, then $p_{m}$ is effectively hyperbolic.

V. Ya Ivriú, V. M. Petkov and L. Hörmander have completely classified the canonical types of $p_{l o c(2)}$ by means of the symplectic transformations to prove the necessity of the Levi condition. They give us good feelings for the structures of hyperbolic operators. So we quote the following from L. Hörmander [7].

Theorem 1.5 (L. Hörmander). Let $Q$ be a quadratic form $p_{\text {!oc(2) }}$ on the symplectic space $V=\boldsymbol{R}^{n+1} \times \boldsymbol{R}^{n+1}$ with $\sigma((x, \xi),(y, \eta))=\langle\xi, y\rangle-\langle x, \eta\rangle$ such that $p_{\text {loc (2) }}(\theta)<0$ and $p_{\text {loc(2) }}$ is hyperbolic with respect to a direction $\theta$. Then $V$ is a direct sum of symplectic subspaces $W_{j}$ which are mutually orthogonal with respect to the symplectic form and $Q$.

Every subspace $W_{j}$ is of one of the following types:

I) $W$ is two dimensional with coordinates $(x, \xi), \sigma((x, \zeta),(y, \eta))=\xi y-x \eta$ and $Q$ has onc of the following forms in $W_{j}$ : a) $Q=\mu\left(x^{2}+\xi^{2}\right), \mu>0$ b) $Q=$ $\mu\left(x^{2}-\xi^{2}\right), \mu>0$ c) $Q=\check{\zeta}^{2}$, d) $Q=-\xi^{2}$, e) $Q=0$.

II) $W$ is four dimensional with coordinates $\left(x_{1}, x_{2}, \xi_{1}, \xi_{2}\right)$ and $\sigma((x, \breve{\zeta})$, 
$(y, \eta))=\langle\xi, y\rangle-\langle x, \eta\rangle$ and $Q=-\xi_{0}^{2}+2 \xi_{0} \xi_{1}+x_{1}^{2}$.

Exactly one of the indefinite cases Ib), Id) and II) occurs, and the decomposition is unique apart from the order of the spaces $W_{j}$, that is, the number of spaces of different types and the numbers $\mu$ are uniquely determind.

According to the above theorem, $p_{\text {loc(2) }}$ can be classified into three types including $\mu\left(x^{2}-\xi^{2}\right),-\xi^{2}$ or $-\xi_{0}^{2}+2 \xi_{0} \xi_{1}+x_{1}^{2}$. An effectively hyperbolic operator corresponds to the case of $\mu\left(x^{2}-\xi^{2}\right)$, and the other cases need the Levi condition. Therefore we can give easily typical examples of hyperbolic operators with double characteristics.

Examples.

1) $-\xi_{0}^{2}+x_{0}^{2}\left(\xi_{1}^{2}+\xi_{2}^{2}\right)+a^{2} x_{1}^{2}\left(\xi_{1}^{2}+\xi_{2}^{2}\right)+b^{2} \xi_{1}^{2} \quad$ on $\quad \boldsymbol{R}^{3}$ is an effectively hyperbolic operator.

2) $-\xi_{0}^{2}+a^{2} x_{1}^{2}\left(\xi_{1}^{2}+\xi_{2}^{2}\right)+b^{2} \xi_{1}^{2} \quad$ on $\boldsymbol{R}^{3}$

and

$$
-\xi_{0}^{2}+2 \xi_{0} \xi_{1}+x_{1}^{2}\left(\xi_{2}^{2}+\xi_{3}^{2}\right)+a^{2} x_{2}^{2}\left(\xi_{2}^{2}+\xi_{3}^{2}\right)+b^{2} \xi_{2}^{2} \quad \text { on } \quad \boldsymbol{R}^{4}
$$

need the Levi conditions bounded by $2^{-1} \operatorname{Tr} \sim \mathscr{F}=|a b|$ at $\xi_{0}=b \xi_{1}=a x_{1}=0$ and at $\xi_{0}=\xi_{1}=b \xi_{2}=x_{1}=a x_{2}=0$, respectively.

\section{§2. Sufficient Conditions}

At the previous section, it has been explained that hyperbolic operators with double characteristic sets are classified into three types. One called effectively hyperbolic has required no limit for lower order terms, and the others have needed some restrictions. We approach to these types from a view point of sufficiency. We restrict the operators treated here to ones of second order for the sake of simplicity because operators with double characteristic set are essentially equivalent to ones of second order with respect to types of characteristic sets.

First, we deal with effectively hyperbolic cases. O. A. Oleinik [23] and others raise to us an idea that the Cauchy problems for effectively hyperbolic operators might be proved to be well posed without any other restrictive condition, because their results have proved so for typical examples. The author has answered to the conjecture by finding a standard type of effectively hyperbolic 
operators. The combination of the author [11] and [12] yields the following theorem.

Definition 2.1. The Cauchy problem for $p$ is said to be $\mathscr{E}$-well posed if it is well posed in the sense of Definition 1.1 and if any solution $u$ there is infinitely differentiable.

Theorem 2.1. Let $p$ be a partial differential operator of second order. If the principal part $p_{2}$ is effectively hyperbolic with respect to $d x_{0}$, then the Cauchy problem for $p$ is $\mathscr{E}$-well posed with respect to $d x_{0}$. The solution $u$ satisfies the estimates, by Sobolev norms on $x_{0}<T$, that $\|u\|_{s} \leq C_{s}\|f\|_{s+l}$ with some constant $l$ independent of $s$, where the support of the datum $f$ lay on a bounded domain.

This result combines with the result by V. Ya Ivrii and V. M. Petkov to yield an assertion.

Corollary 2.2. Let $p_{2}$ be a principal part of a partial differential operator of second order. $p_{2}$ is strongly hyperbolic if and only if $p_{2}$ is effectively hyperbolic.

We mention a standard type of effectively hyperbolic operators, which helps us to understand them.

Theorem 2.3. Any effectively hyperbolic $p_{2}$ with infinitely differentiable coefficients is written locally as

$$
p_{2}=-\left(\xi_{0}-\Lambda_{1}\right)\left(\xi_{0}-\Lambda_{0}\right)+b_{2},
$$

where $\Lambda_{0}, \Lambda_{1}$ and $b_{2}$ are infinitely differentiable functions in $\left(x_{0}, x, \xi\right)$ of homogeneous order 1,1 and 2 in $\xi$, respectively, such that

$$
\begin{gathered}
b_{2} \geq 0, \\
\left\{\xi_{0}-\Lambda_{0}, \xi_{0}-\Lambda_{1}\right\}>0 \text { at } \Lambda_{0}-\Lambda_{1}=b_{2}=0
\end{gathered}
$$

and

$$
\left\{\xi_{0}-\Lambda_{0}, b_{2}\right\}+c b_{2}=0
$$

with an infinitely differentiable function $c$ in $\left(x_{0}, x, \xi\right)$.

The Cauchy problem does not admit all symplectic transformation. It causes some complexities in both proofs of necessary parts and sufficient parts. In fact the above standard type also is not always the canonical type. For ex- 
ample, let us consider

$$
p_{2}=-\tau^{2}+(t \eta+\zeta)^{2}+2^{-1} x^{2} \eta^{2}
$$

The canonical type is

$$
p_{2}=-2^{-1} \sigma^{2}+s^{2} \omega^{2}+\zeta^{2}
$$

by the symplectic transformation such that $\sigma=2 \tau+x \eta, s=t+\xi / \eta, \zeta=\tau+x \eta$, $z=t / 2-\xi_{i}(2 \eta), \omega=\eta$ and $w=y+x \xi / \eta$. The standard type is, however,

$$
\begin{aligned}
& p_{2}=-\tau^{2}+\Lambda_{0}^{2}+b_{2}, \\
& \Lambda_{0}=2^{-1 / 2}\left(t+\check{\zeta} / \eta-2^{-1 / 2} x\right)|\eta|
\end{aligned}
$$

and

$$
b_{2}=2^{-1}\left(1 \eta+\xi+2^{-1 / 2} x \eta\right)^{2},
$$

where they should be properly modified on a conic neighborhood of $\{\eta=0\}$.

The non effectively hyperbolic cases have not yet conditions as clear as in the effectively hyperbolic cases. One of the reasons is caused by the difficulty of development from pointwise situations of conditions to local situations. There are, however, some cases with additional conditions, which are finally reduced to the following according to $\mathrm{V}$. Ya Ivrii [10].

The principal part $p_{2}$ is written with the function $\Lambda_{0}, \Lambda_{1}$ and $b_{2}$ in $\left(x_{0}, x, \check{\zeta}\right)$ of homogeneous order 1,1 and 2 in $\xi$, respectively, as

$$
\begin{gathered}
p_{2}=-\left(\xi_{0}-\Lambda_{1}\right)\left(\xi_{0}-\Lambda_{0}\right)+b_{2}, \\
b_{2} \geq 0
\end{gathered}
$$

and

$$
\left|\left\{\xi_{0}-\Lambda_{0}, b_{2}\right\}\right| \leq C b_{2}
$$

with a positive constant $C$. It is clear that this type of $p_{2}$ is not effectively hyperbolic at any singular point of the characteristic set (double characteristic points) if $\left\{\check{\zeta}_{0}-\Lambda_{0}, \zeta_{0}-\Lambda_{1}\right\}$ vanishes there. As to this type, the following result is easily obtained by virtue of the inequality due to A. Melin [18].

Theorem 2.4(V. Ya Ivriǐ). Let $p_{2}$ be defined as the above. If the positive trace $\operatorname{Tr} \mathscr{F}$ of the fundamental matrix $\mathscr{F}$ do not vanish at the double characteristic points and if the subprincipal part $p_{1}$ is bounded as

$$
\left|\operatorname{Re} p_{1}\right|<2^{-1} \operatorname{Tr}^{\sim} \mathscr{F}
$$

at the double characteristic points and 


$$
\left|\operatorname{lm} p_{1}+2^{-1}\left\{\xi_{0}-\Lambda_{0}, \xi_{0}-\Lambda_{1}\right\}+\theta_{0} \Lambda_{0}+\theta_{1} \Lambda_{1}\right|^{2} \leq C b_{2}
$$

with infinitely differentiable functions $\theta_{j}$ in $\left(x_{0}, x, \xi\right)$ and a constant $C$, then the Cauchy problem for a partial differential operator $p=p_{2}+p_{1}+p_{0}$ is $\mathscr{E}$-well posed. The solution $u$ satisfies the estimates that

$$
\|u\|_{s+1 / 2} \leq C_{s}\|f\|_{s}
$$

for any Sobolev norm on $x_{0}<T$ if the support of datum $f$ is included in a fixed bounded set.

Remark. The examples at the previous section satisfy the conditions of the theorem if the constant $a b$ does not vanish and if the subprincipal parts satisfy them.

Let us apply it to the cases of exactly double characteristics, that is, suppose that the singular points of the characteristic set of $p_{2}$ form a submanifold and the Hesse matrices on it are non-degenerate with respect to vectors transversal to the submanifold. It is important to pick out the term $\xi_{0}-\Lambda_{0}$ from $p_{2}$. It is possible in the cases that the fundamental matrices are always of type Id) of Theorem 1.5 and null eigenspaces of $\mathscr{F}$ have a constant dimension.

Theorem 2.5 (V. Ya Ivriǐ and L. Hörmander). Let $p$ be a partial differential operator such that the principal part $p_{2}$ is hyperbolic with respect to $d x_{0}$, the set of double characteristic points is exactly double, the fundamental matrices on it are always of type Id) and the null eigenspaces have a constant dimension on it. If the subprincipal part $p_{1}$ satisfies, at the double characteristic point, that

$$
-2^{-1}(1-\varepsilon) \operatorname{Tr}^{\sim} \mathscr{F} \leq p_{1} \leq 2^{-1}(1-\varepsilon) \operatorname{Tr} \sim \mathscr{F}
$$

for some positive $\varepsilon$, then the Cauchy problem for $p$ is $\mathscr{E}$-well posed.

Remark. We refered to L. Hörmander [7] for the formulation of this theorem. The case of non-nilpotent $\mathscr{F}$ is due to V. Ya Ivrii [10]. The case of nilpotent $\mathscr{F}$, that is, $\operatorname{Tr} \sim \mathscr{F}=0$, is due to $\mathrm{L}$. Hörmander, which needs the improved A. Melin's inequality with the strong bound, so that it implies only the estimate that $\|u\|_{s} \leq C_{s}\|p u\|_{s}$.

The result by O. A. Oleinik [23] should be stated before finishing this section, because it includes many types with double characteristics not discussed in this article and because it has motivated many related works including V. Ya Ivriǐ and V. M. Petkov's after it. 
Theorem 2.6(O. A. Oleinik). Let $p$ be a partial differential operator such that the principal part $p_{2}$ is $\xi_{0}^{2}-b_{2}\left(x_{0}, x, \xi\right)$, where $b_{2} \geq 0$. The Cauchy problem

$$
p u=f \quad \text { at } \quad 0 \leq x_{0}<T
$$

and

$$
\left.u\right|_{x_{0}=0}=u_{0},\left.\left(\partial / \partial x_{0}\right) u\right|_{x_{0}=0}=u_{1}
$$

for infinitely differentiable data $u_{0}, u_{1}$ and $f$ with compact supports has a unique infinitely differentiable solution $u$ if the subprincipal part $p_{1}$ satisfies

$$
\alpha x_{0}\left(\left.p_{1}\right|_{\xi_{0}=0}\right)^{2} \leq A b_{2}+c\left(\partial / \partial x_{0}\right) b_{2}
$$

at $0 \leq x_{0} \leq T$ with some positive constant $\alpha$ and $A$.

\section{§3. At the End}

We have no concrete and general result at multiple characteristic points. Is it possible to get the canonical types, by means of symplectic transformations, of the principal part of the localization $p_{l o c}$, which is a multi-linear form in $(x, \xi)$ and a hyperbolic polynomial with respect to the direction $\theta \neq 0$ as a polynomial in $(x, \xi)$ ? In the case of double characteristic points, according to L. Hörmander [7], the necessary conditions are closely related to getting a fundamental solution in tempered distributions of the Cauchy problem for the localization, that is, to finding a solution $F(x, y)$ in $\mathscr{S}^{\prime}$ of the equation

$$
p_{l o c}(x, D) F(x, y)=\delta(x-y)
$$

and

$$
F(x, y)=0 \text { if }\langle x-y, \theta\rangle<0 .
$$

Are there any necessary and sufficient conditions of the above problem for the localization of higher order? The localization defined in this article is not general. For example, the necessary and sufficient condition to be well posed for the equation

$$
\tau^{2}-t^{2 k} \xi^{2}+a t^{l} \xi \quad(a \neq 0, \theta=d t) \quad \text { on } \quad \boldsymbol{R}^{2}
$$

is that $l \geq k-1$. We have not discussed these cases $(k>1)$ in this article. The localization of this operator at $t=x=\tau=0$ and $\xi \neq 0$ should be considered as $\tau^{2}-t^{2 k} \xi^{2}$ if $l>k-1, \tau^{2}+a t \xi \xi$ if $l<k-1$ and itself if $l=k-1$. It seems effective according to S. Mizohata [20] and T. Mandai [16] that the weight controlling 
the localization is defined by means of the Newton polygon.

Some questions arise immediately of sufficiency. Under what conditions are the principal part $p_{2}$ able to have a decomposition by smooth functions at Theorem 2.4, especially, in the case of type II) at Theorem 1.5? Are there any methods independent of such a decomposition, especially, a method applying to the case of fixed one of non effectively hyperbolic types and non nilpotent fundamental matrices? The case of nilpotent fundamental matrices at Theorem 2.5 means that the manifold of double characteristic points is involutive. The condition that the subprincipal part vanishes there is equivalent to the condition that $p$ is weaker than $p_{2}$ in the sense by L. Hörmander and L. Svensson. The results by J. L. Dunn and S. Wakabayashi show us the posibility of a condition independent of change of variables unifying the cases of constant coefficients, constant multiplicity and the above as "involutive" cases. If the establishment of such a notion is possible, it will be one of big conditions for the Cauchy problem.

In this article, we mainly dealt with only cases of conditions stated by means of fundamental matrices. There are many related works after O. A. Oleinik. At References 2, we collected some of them published after V. Ya Ivriǐ and V. M. Petkov without comment. (See the detailed references by V. Ya Ivriǐ and V. M. Petkov for works before them.) It would be our great pleasure if an aspect for farther clarifications would be found from among them.

\section{References 1}

[1] Chazarain, J., Opérateurs hyperboliques à caractéristiques de multiplicité constant, Ann. Inst. Fourier, 24 (1974), 173-202.

[2] Chi Min-You, On the Cauchy problem for a class of hyperbolic equations with data given on the degenerate parabolic line, Acta Math. Sinica, 8 (1958), 521-529. (Chinese Math. (1967), 246-254).

[ 3 ] Dunn, J. L., A sufficient condition for hyperbolicity of partial differential operators with constant coefficient principal part, Trans. Amer. Math. Soc., 201 (1975), 315-327.

[4] Flaschka, H. and Strang, G., The correctness of the Cauchy problem, Advances in Math., 6 (1971), 347-379.

[5] Hadamard, J., Le Problème de Cauchy et les Equations aux Dérivées Partielles Linéaires Hyperboliques, Hermann, Paris, 1932.

[6] Hörmander, L., Linear partial differential operators, Springer-Verlag, BerlinGöttingen-Heidelberg, 1963.

[7] — The Cauchy problem for differential equations with double characteristics, J. Analyse Math., 32 (1977), 118-196.

[ 8 ] Gårding, L., Linear hyperbolic partial differential equations with constant coefficients, Acta Math., 85 (1950), 1-62. 
[9] Ivriǐ, V. Ya and Petkov, V. M., Necessary conditions for the Cauchy problem for non-strictly hyperbolic equations to be well-posed, Uspehi Mat. Nauk, 29 (1974), 3-70. (Russian Math. Surveys, 29 (1974), 1-70.)

[10] Ivrii, V. Ya, The well-posedness of the Cauchy problem for nonstrictly hyperbolic operators, Trudy Moskov. Mat. Obsc., 34 (1977), 151-170. (Trans. Moscow Math. Soc. 1978, Issue 2, 149-168).

[11] Iwasaki, N., The Cauchy problem for effectively hyperbolic equations (a special case), J. Math. Kyoto Univ., 23 (1983), 503-562.

[12] - The Cauchy problem for effectively hyperbolic equations (a standard type), Publ. RIMS, Kyoto Univ., 20, to appear.

[13] Lax, A., On Cauchy's problem for partial differential equations with multiple characteristics, Comm. Pure Appl. Math., 9 (1956), 135-169.

[14] Lax, P. D., Asymptotic solutions of osillatory initial value problems, Duke Math. J., 24 (1957), 627-646.

[15] Levi, E. E., Caracteritiche multiple e problema di Cauchy, Ann. Mat. Pura Appl., 16 (1909), 109-127.

[16] Mandai, T., Necessary conditions for well-posedness of the flat Cauchy problem and the regularity-loss of solutions, preprint RIMS-391, RIMS, Kyoto Univ., 1982.

[17] Matsuura, S., On non-strict hyperbolicity, Proc. of Int. Conf. on Functional Anal., Tokyo, (1969), 171-176.

[18] Melin, A., Lower bounds for pseudo-differential operators, Ark. Mat., 9 (1971), $117-140$.

[19] Mizohata, S., Some remarks on the Cauchy problem, J. Math. Kyoto Univ., 1 (1961), 109-127.

[20] — On the Cauchy-Kowalewski theorem, Adv. Math., Suppl. Stud. 7B (1981), $617-652$.

[21] Mizohata, S. and Ohya, Y., Sur la condition de E. E. Levi concernant des équations hyperboliques, Publ. RIMS, Kyoto Univ., 4 (1968/9), 511-526.

[22] — Sur la condition d'hyperbolicité pour les équations à caracteristiques multiples II, Japan J. Math., 40 (1971), 63-104.

[23] Olejnik, O. A., On the Cauchy problem for weakly hyperbolic equations, Comm. Pure Appl. Math., 23 (1970), 569-586.

[24] Petrovskii, I. G., Über das Cauchysche Problem für ein System linearer partieller Differentialgleichungen im Gebiete der nichtanalytischen Funktionen, Bull. Univ. Moscou, Sér. Int. 1 , No. 7 (1938), 1-72.

[25] — Über das Cauchysche Problem für Systeme von partiellen Differentialgleichungen, Mat. Sb., 2 (1937), 815-866.

[26] Svensson, S. L., Necessary and sufficient conditions for the hyperbolicity of polynomials with hyperbolic principal part, Ark. Mat., 8 (1969), 145-162.

[27] Wakabayashi, S., The Cauchy problem for operators with constant coefficient hyperbolic principal part and propagation of singularities, Japan. J. Math., 6 (1980), 179228.

[28] Yamaguti, M., Le problème de Cauchy et les opérateurs d'intégrale singulière, Mem. Coll. Sci. Univ. Kyoto Ser. A, 32 (1959), 121-151.

\section{References 2}

[29] Alinhac, S., A class of hyperbolic operators with double involutive characteristics of Fuchsian type, Comm. in P. D. E., 3 (1978), 877-905. 
[30] - Branching of singularities for a class of hyperbolic operators, Indiana Univ. Math. J., 27 (1978), 1027-1037.

[31] — - Paramétrix et propagation des singularités pour un problème de Cauchy à multiplicité variable. Asterisque, 34-35 (1976), 3-26.

[32] - Paramétrix pour un système hyperbolique à multiplicité variable, Comm. in P. D. E., 2 (1977), 251-296.

[33] _ _ Solution explicite du problème de Cauchy pour des opérateurs effectivement hyperboliques, Duke Muth. J., 45 (1978), 225-258.

[34] Amano, K. and Nakamura, G., Branching of singularities for degenerate hyperbolic operators and Stokes' phenomena II, Proc. Japan Acad., 57 (1981), 164-167.

[35] Amano, K., Branching of singularities for degenerate hyperbolic operators and Stokes' phenomena, Proc. Japan Acad., 56 (1980), 206-209.

[36] Bersin, R. et Vaillant, J., Paramétrix du problèmes de Cauchy pour un système faiblement hyperbolique à caractéristiques multiples, C. R. Acad. Sci. Paris, 283 (1976), 485-487.

[37] - Systèmes hyperboliques á caractéristiques multiples, J. Math. Pures et Appl.. 58 (1979), 165-216.

[38] Colombini. F. and Spagnolo, S., An example of a weakly hyperbolic Cauchy problem not well posed in $C^{\infty}$, Acta Math., 148 (1982), 243-253.

[39] - On the convergence of solutions of hyperbolic equations, Comm. in P. D. E., 3 (1978), 77-103.

[40] Colombini, F., De Giorgi, E. and Spagnolo, S., Sur les équations hyperboliques avec des coefficients qui ne dépendent que du temps. Ann. Scuola Norm. Sup. Pisa, 6 (1979), 511-559.

[41] Demay, Y.. Le problème de Cauchy pour les systèmes hyperboliques à caractéristiques doubles, C. R. Acad. Sci. Paris, 278 (1974), 771-773.

[42] ——, Paramétrix pour des systènes hyperboliques du premier order à multiplicité constante, J. Math. Pure Appl., 56 (1977), 393-422.

[43] Gourdin, D., Les opérateurs faiblement hyperboliques matriciels à caractéristiques de multiplicités constantes, bien decomposables et le problème de Cauchy non caractéristique associé, J. Math. Kyoto Univ., 17 (1977), 539-566.

[44] — Problème de Cauchy pour des systèmes hyperboliques à caractéristiques de multiplicité variable, C. R. Acad. Sci. Paris, 292 (1981), 789-791.

[45] — Systèmes faiblement hyperboliques à caractéristique multiples, $C . R$. Acad. Sci. Paris, 278 (1974), 269-273.

[46] Hanges, N., Parametrices and propagation of singularities for operators with noninvolutive characteristics, Indiana Univ. Math. J., 28 (1979), 87-97.

[47] — Parametrices and local solvability for a class of singular hyperbolic operators, Comm. in P. D. E., 3 (1978), 105-152.

[48] Ichinose, W. and Kumano-go, H., On the propagation of singularities with infinitely many branching points for a hyperbolic equation of second order, Comm. in P. D. E., 6 (1981), 569-623.

[49] Ichinose, W., Propagation of singularities for a hyperbolic equation with non-regular characteristic roots, Osaka J. Math., 17 (1980), 703-749.

[50] Igari, K., A necessary condition for well-posed Cauchy problems, J. Math. Kyoto Univ., 16 (1976), 531-543.

[51] — Cauchy problem for weakly hyperbolic equations of second order, $J$. Math. Kyoto Univ., 20 (1980), 43-55.

[52] Imai, M., On a parametrix for a weakly hyperbolic operator, Hokkaido Math. J., 9 (1980), 190-216. 
[53] Ivriǐ, V. Ya, Energy integrals for non-strictly hyperbolic operators, Uspekhi Mat. Nauk., 30: 6 (1975), 169-170.

[54] — Sufficient conditions for regular and completely regular hyperbolicity, Trudy Moscov Obsc., 33 (1976), 3-66. (Transaction of Moscow Math. Soc., 33 (1978), 1-65.)

[55] - Wave fronts of solutions of certain pseudo-differential equations, Fun. Anal. Appl., 10 (1976), 141-142.

[56] — Wave fronts of solutions to some microlocally hyperbolic pseudo-differential equations, Dokl. Akad. Nauk USSR, 226 (1976), 1257-1259, Soviet Math. Dokl., 17 (1976), 233-236.

[57] — Wave fronts of solutions to some pseudo-differential equations, Moskov. Mat. obsc., 39 (1979), 49-82.

[58] Iwasaki, C. and Morimoto, Y., Propagation of singularities of solutions for a hyperbolic system with nilpotent characteristics. I, Comm. in P. D. E., 7 (1982), 743-794.

[59] John, F., Restrictions on the coefficients of hyperbolic systems of partial differential equations, Proc. Nat'l Acad. Sci. USA, 74 (1977), 4150-4151.

[60] - Algebraic conditions for hyperbolicity of systems of partial differential equations, Comm. Pure. Appl. Math., 31 (1978), 89-106, 787-793.

[61] Kajitani, K., Cauchy problem for non-strictly hyperbolic systems, Publ. RIMS, Kyoto Univ., 15 (1979), 519-550.

[62] - Cauchy problem for non-strictly hyperbolic systems II, Leray-Volevich's systems and well-posedness, J. Math. Kyoto Univ., 22 (1982), 261-283.

[63] Kitagawa, K. and Sadamatsu, T., Sur une condition suffisante pour que le problème de Cauchy faiblement hyperbolique soit bien posé - Cas de multiplicité de caracteristiques au plus triple, J. Math. Kyoto Univ., 17 (1977), 465-499.

[64] Kumano-go, H. and Taniguchi, K., Fourier integral operators of multi-phase and the fundamental solution for a hyperbolic system, Funkcialaj Ekvacioj, 22 (1979), 161-196.

[65] Kumano-go, H., A calculus of Fourier integral operators on $\boldsymbol{R}^{n}$ and the fundamental solution for an operator of hyperbolic type, Comm. in P. D. E., 1 (1976), 1-44.

[66] — - Fundamental solution for a hyperbolic system with diagonal principal part, Comm. in P. D. E., 4 (1979), 959-1015.

[67] — Fundamental solutions for operators of regularly hyperbolic type, $J$. Math. Soc. Japan, 29 (1977), 399-406.

[68] Kumano-go, H., Taniguchi, K. and Tozaki, Y., Multi-products of phase functions for Fourier integral operators with an application, Comm. in P.D.E., 3 (1978), 349-380.

[69] Lascar, B., Propagations des singularités pour des équations hyperboliques à caractéristique de multiplicité au plus double et singularités Masloviennes, Amer. J. Math., 104 (1982), 227-285.

[70] Lascar, B. and Lascar, R., Propagations des singularités pour des équations hyperboliques à caractéristique de multiplicité au plus double et singularités Masloviennes, II, J. Anal. Math., 41 (1982), 1-38.

[71] Mandai, T., A necessary and sufficient condition for the well-posedness of some weakly hyperbolic Cauchy problems, preprint RIMS-404, RIMS, Kyoto Univ., 1982.

[72] — On energy inequalities and regularity of solutions to weakly hyperbolic Cauchy problems, Publ. RIMS, Kyoto Univ., 18 (1982), 187-240.

[73] Matsumoto, W., On the conditions for hyperbolicity of systems with double characteristic roots, I, II, J. Math. Kyoto Univ., 21 (1981), 47-84, 251-271.

[74] — - Sur l'espace de données admissibles dans le problème de Cauchy, $C . R$. 
Acad. Sci. Paris, 292 (1981), 621-623.

[75] Matsuura, S., On the propagation of support of solutions to general systems of partial differential equations, Lect. Notes in Phys., 39 (1975), 380-386.

[76] Menikoff, A., The Cauchy problem for weakly hyperbolic equations, Amer. $J$. Math., 97 (1975), 548-558.

[77] Morimoto, Y., Fundamental solution for a hyperbolic equation with involutive characteristics of variable multiplicity, Comm. in P. D. E., 4 (1979), 609-643.

[78] Nakamura, G. and Uryu, H., Parametrix of certain weakly hyperbolic operators, Comm. in P. D. E., 5 (1980), 837-896.

[79] Nishitani, T., A necessary and sufficient condition for the hyperbolicity of second order equations with two independent variables, preprint, 1982.

[80] - On the Cauchy problem for weakly hyperbolic equations, Comm. in P. D. E., 3 (1978), 319-333.

[81] - Some remarks on the Cauchy problem for weakly hyperbolic equations, J. Math. Kyoto Univ., 17 (1977), 245-258.

[82] - The Cauchy problem for weakly hyperbolic equations of second order, Comm. in P. D. E., 5 (1980), 1272-1296.

[83] Nishiwada, K., On necessary conditions for Cauchy problems to be microlocally well posed, Japan. J. Math., 6 (1980), 1-44.

[84] Nosmas, J. C., Paramétrix du problème de Cauchy pour une classe de systèmes hyperboliques symétrisables à caractéristiques involutives de multiplicité variable, Comm. in P. D. E., 5 (1980), 1-22.

[85] —- Paramétrix du problème de Cauchy pour une classe d'opérateurs à caractéristiques de multiplicité variable, C.R. Acad. Sci. Paris, 285 (1977), 1065-1068.

[86] Ohya, Y., Le problème de Cauchy à caractèristique multiples, Ann. Scuola Norm. Sup. Pisa, 4 (1977), 757-805.

[87] — Le problème de Cauchy à caractéristiques multiples - méthode directe pour obtenir la condition (généralisée) de E. E. Levi, C. R. Acad. Sci. Paris, 282 (1976), 1433-1436.

[88] Petkov, V. M., The Cauchy problem for a class of non-strictly hyperbolic equations with double characteristics, Serdica, Bulg. Mat. Publ., 1 (1975), 372-380.

[89] - The parametrix of the Cauchy problem for non symmetrizable hyperbolic systems with characteristics of constant multiplicity, Tr. Mosk. Mat. O.-va, 37 (1978), 3-47. (Trans. Mosc. Math. Soc., 37 (1980), 1-47.)

[90] — Une parametrix du problème de Cauchy pour des systèms hyperboliques à caractéristiques de multiplicité constante, C. R. Acad. Sci. Burgare, 29 (1976), 10951097.

[91] Sakamoto, R., Cauchy problem for degenerate hyperbolic equations, Comm. Pure Appl. Math., 33 (1980), 785-816.

[92] — On a sufficient condition for well-posedness of weakly hyperbolic Cauchy problems, Publ. RIMS, Kyoto Univ., 15 (1979), 469-518.

[93] — Some remarks on degenerate hyperbolic Cauchy problems, Comm. Pure Appl. Math., 33 (1980), 817-830.

[94] Shinkai, K., Branching of singularities for a degenerate hyperbolic system, Comm. in P. D. E., 7 (1982), 581-607.

[95] - Fundamental solution of a degenerate hyperbolic system, Osaka $J$. Math., 18 (1981), 257-288.

[96] Steinberg, S., Existence and uniquenesss of the solutions of hyperbolic equations which are not necessarily strictly hyperbolic, J. Diff. Eq., 17 (1975), 119-153. 
[97] Tahara, H., Singular hyperbolic systems, I, II, III, J. Fac. Sci. Univ. Tokyo, Sec. IA, 26 (1979), 213-238, 391-412: 27 (1980), 465-507.

[98] Takasaki, K., Singular Cauchy problems for a class of weakly hyperbolic differential operators, Proc. Japan Acad., 57 (1981), 393-397.

[99] Taniguchi, K. and Tozaki, Y., A hyperbolic equation with double characteristics which has a solution with branching singularities, Math. Japonica. 25 (1980), 279-300.

[100] Tarama, S., Le problème de Cauchy pour une classe des systèmes faiblement hyperboliques, Comm. in P. D. E., 4 (1979), 809-825.

[101] — Un exemple dans le problème de Cauchy pour les équations faiblement hyperboliques, Publ. RIMS. Kyoto Univ., 15 (1979), 455-468.

[102] Uhlmann, G. A., Parametrices for operators with multiple involutive characteristics, Comm. in P. D. E., 4 (1979), 739-767.

[103] - Pseudodifferential operators with double involutive characteristics, Comm. in P. D. E., 2 (1977), 713-779.

[104] Uryu, H., The Cauchy problem for weakly hyperbolic equations, Comm. in P. D. E., 5 (1980), 23-40.

[105] — The Cauchy problem for weakly hyperbolic systems with variable multiple characteristics, Publ. RIMS, Kyoto Univ., 15 (1979), 719-739.

[106] - The Cauchy problem for weakly hyperbolic equations. II: Infinite degenerate case, Tokyo J. Math., 3 (1980), 99-113.

[107] Yagdzhyan, K. A., On the correctness of the Cauchy problem for weakly hyperbolic equations, Izu. Akad. Nauk Arm. SSR, Mat. 15 (1981), 475-487.

[108] Yamahara, H., On the Cauchy problems for weakly hyperbolic systems, Publ. RIMS, Kyoto Univ., 12 (1976), 493-512.

[109] Yamamoto, K., Some examples of the $C^{\infty}$ well-posed Cauchy problem, J. Lond. Math. Soc., II. ser. 22 (1980), 307-317.

[110] - The Cauchy problem for some class of hyperbolic differential operators with variable multiple characteristics, J. Math. Soc. Japan, 31 (1979), 481-502.

[111] Yoshikawa, A., Construction of a parametrix for the Cauchy problem of some weakly hyperbolic equation, I, II, III, Hokkaido Math. J., 6 (1977), 313-344, 7 (1978), 1-26, 127-141.

[112] - Fundamental solutions to the Cauchy problem of some weakly hyperbolic equation, Proc. Japan Acad., 53 (1977), 103-107.

[113] - Parametrices for a class of effectively hyperbolic operators, Comm. in P. D. E., 5 (1980), 1073-1151.

[114] Zajtseva, O. V., On the correctness of the Cauchy problem for certain degenerate hyperbolic equations, Izv. Vyssh. Uchebn. Zaved., Mat. No. 5 (1980), 83-84. (Sov. Math., 24 No. 5 (1980), 99-101.)

[115] - On the correctness of a Cauchy problem for a model equation with fourfold characteristics, Izv. Vyssh. Uchebn. Zaved., Mat. No. 6 (1980), 22-22. (Sov. Math., 24 No. 6 (1980), 21-23.)

[116] Zeman, M., On the generalized Levi condition for partial differential operators with double characteristics, Comm. in P. D. E., 3 (1978), 1041-1052.

[117] - The Levi-Lax condition for partial differential equations with real characteristics of constant multiplicity, Isrciel J. Math., 31 (1978), 57-77.

[118] - The well-posedness of the Cauchy problem for partial differential equations with multiple characteristics, Comm. in P. D. E., 2 (1977), 223-249. 